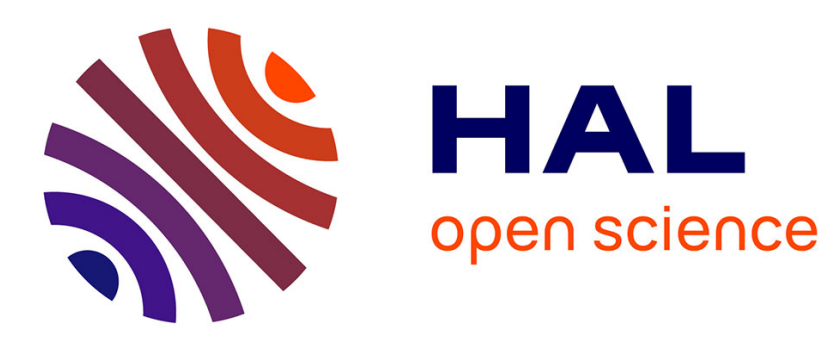

\title{
Renormalisation group study of a disordered model
}

Bernard Derrida, E. Gardner

\section{To cite this version:}

Bernard Derrida, E. Gardner. Renormalisation group study of a disordered model. Journal of Physics A : Mathematical and General, 1984, 17 (16), pp.3223-3236. 10.1088/0305-4470/17/16/023 . hal03285928

\section{HAL Id: hal-03285928 \\ https://hal.science/hal-03285928}

Submitted on 21 Jul 2021

HAL is a multi-disciplinary open access archive for the deposit and dissemination of scientific research documents, whether they are published or not. The documents may come from teaching and research institutions in France or abroad, or from public or private research centers.
L'archive ouverte pluridisciplinaire HAL, est destinée au dépôt et à la diffusion de documents scientifiques de niveau recherche, publiés ou non, émanant des établissements d'enseignement et de recherche français ou étrangers, des laboratoires publics ou privés. 


\title{
Renormalisation group study of a disordered model
}

\author{
B Derrida and E Gardner \\ SPhT Orme des Merisiers, CEN Saclay, 91191 Gif sur Yvette, Cedex, France
}

Received 11 April 1984

\begin{abstract}
We study the Potts model on a diamond hierarchical lattice with random interactions. Using weak disorder expansions, we calculate analytically the position and the exponents of the random fixed point which appears when the specific heat exponent $\alpha_{p}$ of the pure system becomes positive. At $\alpha_{p}=0$, we find how the logarithmic singularity is modified by the disorder. Lastly we suggest that this model should present Griffiths-like singularities.
\end{abstract}

\section{Introduction}

In the last ten years, real space renormalisation methods have been used to study an increasing number of problems in statistical physics (for a recent review see Burkhardt and Van Leeuwen 1982). Among all the real space renormalisation schemes, a very simple one is the Migdal-Kadanoff method (Migdal 1976, Kadanoff 1976). For models defined on Bravais lattices, the Migdal-Kadanoff renormalisation method is an approximate way of calculating critical points and critical exponents which does not usually give very accurate estimations. However, although it is very simple, one believes that it contains several important features which are common to all the real space renormalisation methods. Moreover, it was realised that one can construct some scale-invariant lattices (called hierarchical lattices) on which the Migdal-Kadanoff method gives an exact renormalisation transformation (Berker and Ostlund 1979). This motivated recently several works (Kaufman and Griffiths 1981, 1983, Griffiths and Kaufman 1982, Melrose 1983, Derrida et al 1983, McKay and Berker 1984, Kaufman and Andelman 1984 ) in particular to distinguish between the features which are peculiar to hierarchical lattices and those which should remain valid on usual Bravais lattices.

The idea of using the Migdal-Kadanoff approximation to study disordered systems is not a new one (Jayaprakash et al 1978, Southern et al 1979, Yeomans and Stinchcombe 1979, Kinzel and Domany 1981, Benyoussef and Boccara 1982). The simplest models of disordered systems are defined by a probability distribution of the nearestneighbour interactions. Using the Migdal-Kadanoff method, one can write the equation which gives the way that this probability distribution is renormalised. Since the space of probability distributions is an infinite-dimensional space, trying to find a fixed point is not an easy mathematical problem (a very simplified example was recently solved by Collet et al 1983). Until now this difficulty has usually been solved by replacing the renormalised distribution by a distribution which belongs to a finite-dimensional space. For example, one decides to consider only distributions which are sums of two delta functions and one replaces the renormalised distribution by the sum of two delta functions which has the same first moments. It is not easy to estimate the accuracy 
of this additional approximation and to know whether some effects are lost. Recently Andelman and Berker (1984) decided to go beyond this approximation and to determine the true fixed point distribution by numerical methods. Although they could obtain interesting estimations of the specific heat exponent $\alpha$ and of the cross over exponent $\phi$ in several situations, their work shows clearly that it is not easy to find accurate values of these exponents.

In the present work, we study the Potts model on a diamond hierarchical lattice with random interactions (Kinzel and Domany 1981, Andelman and Berker 1984). When the specific heat exponent $\alpha_{\mathrm{p}}$ of the pure system becomes positive (this corresponds to $q>q_{0}=4+2 \sqrt{2}$ ) we shall see that the disorder becomes relevant (Harris 1974) and a new fixed point appears in the probability distribution space. In the following we shall call this fixed point, the random fixed point. Using weak disorder expansions, we calculate analytically the location of this random fixed point and of its exponents in powers of $q-q_{0}$. When $\alpha_{\mathrm{p}}=0$, we obtain the way that the specific heat singularity is modified by a narrow distribution of interactions. Lastly, we suggest that this model presents Griffiths (1969) like singularities.

The paper is organised as follows. In $\S 2$, we introduce the model. We describe some properties of the pure system in $\S 3$. In $\S 4$, we give the result of our weak disorder expansion. In $\S 5$ we obtain the fixed points and their exponents for $q-q_{0} \ll 1$. In $\S 6$, we find the equation of the critical surface using the nonlinear scaling fields (Wegner 1976). In $\S 7$, we study in more detail the case where $\alpha_{\mathrm{p}}=0$ and we find the singular part of the specific heat for a narrow distribution of interactions. In $\S 8$, we discuss the possibility of Griffiths singularities.

\section{Definition of the model}

Throughout this paper we consider a Potts model on a diamond hierarchical lattice with random nearest-neighbour interactions. The diamond hierarchical lattice is constructed according to the recursion rule shown in figure 1. On this lattice, we consider a random $q$ state Potts model whose Hamiltonian is

$$
\mathscr{H}=-\sum_{\langle i j\rangle} J_{i j} \delta_{\sigma_{i} \sigma}
$$

where the spins $\sigma_{i}$ can take $q$ different values, the sum runs over all pairs of nearest neighbours on the hierarchical lattice and the interactions $J_{i j}$ are distributed according to a given probability distribution. It will be more convenient to work with Boltzmann

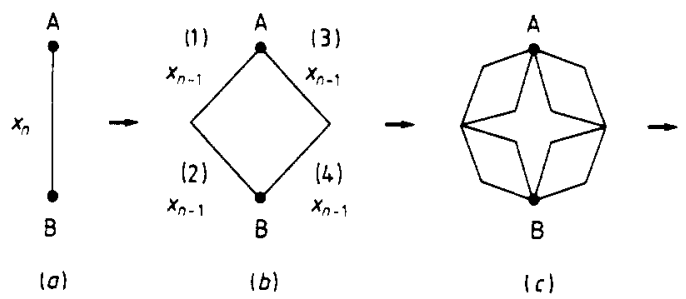

Figure 1. The diamond hierarchical lattice is constructed recursively. One starts with one bond $(a)$. To go from $(a)$ to $(b)$ one replaces this bond by a set of four bonds. To go from $(a)$ to $(c)$ one replaces each bond in $(b)$ by a set of four bonds, and so on. 
factors. Therefore, on each bond we consider a variable $x$

$$
x=\mathrm{e}^{\beta J_{1 j}}
$$

and we consider as given the probability distribution $P_{0}(x)$ of $x$.

Under the decimation procedure, each set of four bonds $x_{n-1}^{(1)}, x_{n-1}^{(2)}, x_{n-1}^{(3)}, x_{n-1}^{(4)}$ at generation $n-1$ can be replaced by one single bond $x_{n}$ at generation $n$ and the renormalisation transformation $R$ is just

$$
\begin{aligned}
x_{n} & =R\left(x_{n-1}^{(1)}, x_{n-1}^{(2)}, x_{n-1}^{(3)}, x_{n-1}^{(4)}\right) \\
& =\left(\frac{x_{n-1}^{(1)} x_{n-1}^{(2)}+q-1}{x_{n-1}^{(1)}+x_{n-1}^{(2)}+q-2}\right)\left(\frac{x_{n-1}^{(3)} x_{n-1}^{(4)}+q-1}{x_{n-1}^{(3)}+x_{n-1}^{(4)}+q-2}\right) .
\end{aligned}
$$

Let us consider a hierarchical lattice of $2\left(4^{N-1}+2\right) / 3$ sites (i.e. $4^{N-1}$ bonds) for which the $x$ have been obtained randomly according to a probability distribution $P_{0}(x)$. After $n$ decimations, we obtain a lattice of $2\left(4^{N-n-1}+2\right) / 3$ sites $\left(4^{N-n-1}\right.$ bonds $)$ whose interactions $x$ are distributed according to a renormalised probability distribution $P_{n}(x)$ which can be deduced from $P_{0}(x)$ using the following recursion formula

$$
P_{n}(x)=\iiint \int \prod_{i=1}^{4}\left(P_{n-1}\left(x_{i}\right) \mathrm{d} x_{i}\right) \delta\left(x-R\left(x_{1}, x_{2}, x_{3}, x_{4}\right)\right) .
$$

If we define the reduced free energy $f\left(\left\{P_{0}\right\}\right)$ per bond by

$$
f\left(\left\{P_{0}\right\}\right)=\lim _{N \rightarrow \infty} \frac{1}{4^{N}} \overline{\log Z_{N}}
$$

where $Z_{N}$ is the partition function of a hierarchical lattice of $4^{N}$ bonds and the average $\overline{\log Z_{N}}$ is performed over all the choices of the nearest-neighbour interactions, then one has

$$
f\left(\left\{P_{0}\right\}\right)=\sum_{n=0}^{\infty} \frac{1}{4^{n}} \iint \mathrm{d} x_{1} \mathrm{~d} x_{2} P_{n}\left(x_{1}\right) P_{n}\left(x_{2}\right) \frac{1}{2} \log \left(x_{1}+x_{2}+q-2\right) .
$$

The problem of finding the phase diagram and the transition lines of this disordered model is equivalent to the problem of finding the surfaces in the space of all the distributions $P_{0}$ where the free energy $f\left(P_{0}\right)$ given by $(6)$ is singular. In $\S 4$, we shall use weak disorder expansion to study this problem. Before doing so, let us recall briefly in $\$ 3$ some properties of the pure model.

\section{The pure system}

By definition, for the pure system, all the interactions $x$ are the same. Therefore the distribution $P_{0}(x)$ is a delta function concentrated at a given point $x_{0}$. Obviously this property remains after decimation and the distributions $P_{n}(x)$ are delta functions

$$
P_{n}(x)=\delta\left(x-x_{n}\right)
$$

The $x_{n}$ are obtained from $x_{0}$ by the following renormalisation transformation $T$ :

$$
x_{n}=R\left(x_{n-1}, x_{n-1}, x_{n-1}, x_{n-1}\right)=T\left(x_{n-1}\right)=\left(\frac{x_{n-1}^{2}+q-1}{2 x_{n-1}+q-2}\right)^{2} .
$$


For each value of $q$, the fixed point $x_{c}$ of the renormalisation transformation is the critical point and $x_{\mathrm{c}}$ is related to $q$ by

$$
q=\left(\sqrt{x_{\mathrm{c}}}-1\right)\left(x_{\mathrm{c}}-1\right) \text {. }
$$

It is easier to express $q$ as a function of $x_{c}$. Therefore in the following, we shall always vary $x_{c}$ instead of $q$, keeping in mind that $q$ is related to $x_{c}$ by (9).

For a given value of the interaction $x_{0}$, the reduced free energy $f\left(x_{0}\right)$ per bond is given by the following series

$$
f\left(x_{0}\right)=\sum_{n=0}^{\infty} \frac{1}{4^{n}} g\left(x_{n}\right)
$$

where $g(x)$ is given by

$$
g(x)=\frac{1}{2} \log (2 x+q-2)
$$

and $x_{n}$ is the $n$th iterate of $x_{0}$ by the transformation $T$.

It is easy to find the singular part of $f$ by noticing that it obeys the following equation

$$
f(x)=\frac{1}{4} f(T(x))+g(x) .
$$

Since in (12), the function $g(x)$ has no singularity at $x_{\mathrm{c}}$, the singular parts coming from $f$ in the two sides of equation (12) must be equal. This implies that the singular part $f_{\text {sing }}$ of $f$ at $x_{\mathrm{c}}$ has the following form

$$
f_{\mathrm{sing}}(x) \sim\left|x-x_{\mathrm{c}}\right|^{2-\alpha_{\mathrm{p}}}\left(\log \left(x-x_{\mathrm{c}}\right) / \log \left(T^{\prime}\left(x_{\mathrm{c}}\right)\right)\right)
$$

where $h$ is a periodic function of period 1 and the specific heat exponent $\alpha_{p}$ of the pure system is given by

$$
\alpha_{\mathrm{p}}=2-\frac{\log 4}{\log \left(T^{\prime}\left(x_{\mathrm{c}}\right)\right)}=2-\frac{\log 4}{\log \left[4 x_{\mathrm{c}} /\left(\sqrt{x_{\mathrm{c}}}+1\right)^{2}\right]} .
$$

The calculation of the periodic function $h$ is not, in general, easy and to our knowledge, only approximate methods of calculating it are known (Derrida et al 1984). However when $\alpha_{\mathrm{p}}$ vanishes one can show that the specific heat has a logarithmic singularity at the critical point and one can calculate the coefficient of the logarithm.

Let us now find the amplitude of this logarithm. From (14), it is easy to see that $\alpha_{\mathrm{p}}$ vanishes for $\sqrt{x_{\mathrm{c}}}=1+\sqrt{2}$, i.e. for

$$
q=4+2 \sqrt{2} \text {. }
$$

Differentiating (12) twice with respect to $x$ gives

$$
f^{\prime \prime}(x)=\frac{1}{4}\left(T^{\prime}(x)\right)^{2} f^{\prime \prime}(T(x))+\frac{1}{4} T^{\prime \prime}(x) f^{\prime}(T(x))+g^{\prime \prime}(x) .
$$

In the neighbourhood of $x_{c}$, using the fact that $T^{\prime}\left(x_{c}\right)=2$, one can replace (16) by

$$
f^{\prime \prime}\left(x_{\mathrm{c}}+x-x_{\mathrm{c}}\right)=f^{\prime \prime}\left(x_{\mathrm{c}}+2\left(x-x_{\mathrm{c}}\right)\right)+\frac{1}{4} T^{\prime \prime}\left(x_{\mathrm{c}}\right) f^{\prime}\left(x_{\mathrm{c}}\right)+g^{\prime \prime}\left(x_{\mathrm{c}}\right)
$$

to find the singular part $f_{\text {sing }}^{\prime \prime}(x)$ of $f^{\prime \prime}$. The general solution to (17) is

$$
f_{\text {sing }}^{\prime \prime}(x)=C \log \left|x-x_{\mathrm{c}}\right|+H\left(\log \left|x-x_{\mathrm{c}}\right| / \log 2\right)
$$

where the constant $C$ is given by

$$
C=-\left(\frac{1}{4} T^{\prime \prime}\left(x_{\mathrm{c}}\right) f^{\prime}\left(x_{\mathrm{c}}\right)+g^{\prime \prime}\left(x_{\mathrm{c}}\right)\right) / \log 2
$$


and $H$ is a periodic function of period 1 in $\log \left|x-x_{\mathrm{c}}\right| / \log 2$. From (8), (11), (12) and (15), one can calculate $T^{\prime \prime}\left(x_{\mathrm{c}}\right), f^{\prime}\left(x_{\mathrm{c}}\right)$ and $g^{\prime \prime}\left(x_{\mathrm{c}}\right)$ and one gets

$$
C=-(1 / 2 \log 2)(29 \sqrt{2}-41)=-(1 / 2 \log 2)(\sqrt{2}-1)^{5} \text {. }
$$

\section{The weak disorder expansion}

In this part, we show how a narrow distribution concentrated around the fixed point $x_{c}$ of the pure system is transformed by one step of the renormalisation transformation (4).

Writing

$$
x_{n-1}^{(i)}=x_{\mathrm{c}}+\varepsilon_{n-1}^{(i)}, \quad x_{n}=x_{\mathrm{c}}+\varepsilon_{n}
$$

we may expand $\varepsilon_{n}$ in powers of the $\varepsilon_{n-1}^{(1)}$ using formula (3). This is what we have done and we have obtained the expression of $\varepsilon_{n}$ in terms of the $\varepsilon_{n-1}^{(1)}$ up to the 4th power. We do not give this expression here because it is rather long and complicated although there is no difficulty in obtaining it from the recursion formula (3).

In the following, we shall consider a special class of narrow distributions. The interest of this class is that when the specific heat exponent $\alpha_{\mathrm{p}}$ of the pure system is small, we shall be able to find a fixed distribution of the renormalisation transformation (4) in this class.

Let us consider the class of narrow distributions which have the following property. We assume that we have at our disposal a small parameter $\lambda$ :

$$
\lambda \ll 1
$$

and we consider narrow distributions such that for $p \geqslant 1$

$$
\overline{\varepsilon^{2 p-1}} \sim \overline{\varepsilon^{2 p}} \sim \lambda^{p} \text {. }
$$

Using the expansion of $\varepsilon_{n}$ in powers of the $\varepsilon_{n-1}^{(1)}$ one can calculate the moments $\overline{\varepsilon_{n}^{p}}$ as functions of the moments $\overline{\varepsilon_{n-1}^{p}}$ up to a given power of $\lambda$. One can easily check that if the moments $\overline{\varepsilon_{n-1}^{p}}$ satisfy condition (23), then the moments $\overline{\varepsilon_{n}^{p}}$ of the renormalised distribution satisfy also condition (23).

We have derived the following equations for the first four moments of the distribution of the $\varepsilon_{n}$ up to the order $\lambda^{2}$.

$$
\begin{aligned}
\overline{\varepsilon_{n}}=4 A \sqrt{x_{\mathrm{c}}} \overline{\varepsilon_{n-1}} & -4 A B \sqrt{x_{\mathrm{c}}} \overline{\varepsilon_{n-1}^{2}}+\left(2 B \sqrt{x_{\mathrm{c}}}-4 A B \sqrt{x_{\mathrm{c}}}+4 A^{2}\right)\left(\overline{\varepsilon_{n-1}}\right)^{2} \\
& +\left(-4 B^{2} \sqrt{x_{\mathrm{c}}}+12 A B^{2} \sqrt{x_{\mathrm{c}}}-8 A^{2} B\right) \overline{\varepsilon_{n-1}} \overline{\varepsilon_{n-1}^{2}} \\
& +\left(4 B^{3} \sqrt{x_{\mathrm{c}}}-12 A B^{3} \sqrt{x_{\mathrm{c}}}+4 A^{2} B^{2}\right)\left(\overline{\varepsilon_{n-1}^{2}}\right)^{2} \\
& +\left(4 A B^{2} \sqrt{x_{\mathrm{c}}}\right) \overline{\varepsilon_{n-1}^{3}}+\left(-4 A B^{3} \sqrt{x_{\mathrm{c}}}\right) \overline{\varepsilon_{n-1}^{4}} \\
\overline{\varepsilon_{n}^{2}}=4 A^{2} x_{\mathrm{c}} \overline{\varepsilon_{n-1}^{2}} & +12 A^{2} x_{\mathrm{c}}\left(\overline{\varepsilon_{n-1}}\right)^{2}+\left(8 A B x_{\mathrm{c}}-40 A^{2} B x_{\mathrm{c}}+16 A^{3} \sqrt{x_{\mathrm{c}}}\right) \overline{\varepsilon_{n-1}} \overline{\varepsilon_{n-1}^{2}} \\
& +\left(2 B^{2} x_{\mathrm{c}}-16 A B^{2} x_{\mathrm{c}}+44 A^{2} B^{2} x_{\mathrm{c}}-16 A^{3} B \sqrt{x_{\mathrm{c}}}+4 A^{4}\right)\left(\overline{\varepsilon_{n-1}^{2}}\right)^{2} \\
& -8 A^{2} B x_{\mathrm{c}} \overline{\varepsilon_{n-1}^{3}}+12 A^{2} B^{2} x_{\mathrm{c}} \overline{\varepsilon_{n-1}^{4}} \\
\overline{\varepsilon_{n}^{3}}=36 A^{3} x_{\mathrm{c}}^{3 / 2} & \overline{\varepsilon_{n-1}} \overline{\varepsilon_{n-1}^{2}}+\left(12 A^{2} B x_{\mathrm{c}}^{3 / 2}-60 A^{3} B x_{\mathrm{c}}^{3 / 2}+24 A^{4} x_{\mathrm{c}}\right)\left(\overline{\varepsilon_{n-1}^{2}}\right)^{2} \\
+ & 4 A^{3} x_{\mathrm{c}}^{3 / 2} \overline{\varepsilon_{n-1}^{3}}-12 A^{3} B x_{\mathrm{c}}^{3 / 2} \overline{\varepsilon_{n-1}^{4}} \\
\overline{\varepsilon_{n}^{4}} & =36 A^{4} x_{\mathrm{c}}^{2}\left(\overline{\varepsilon_{n-1}^{2}}\right)^{2}+4 A^{4} x_{\mathrm{c}}^{2} \overline{\varepsilon_{n-1}^{4}}
\end{aligned}
$$


where $A$ and $B$ are constants given by

$$
A=\sqrt{x_{c}} /\left(\sqrt{x_{c}}+1\right)^{2}, \quad B=1 /\left[\left(x_{c}-1\right)\left(\sqrt{x_{c}}-1\right)\right] .
$$

The only talent required to obtain expressions (24) to (27) is virtuosity in Taylor expansions.

One can also calculate the moments $\overline{\varepsilon_{n}^{2 p-1}}$ and $\overline{\varepsilon_{n}^{2 p}}$ to leading order in $\lambda$, i.e. to order $\lambda^{p}$ and one finds for $p \geqslant 2$

$$
\begin{aligned}
\overline{\varepsilon_{n}^{2 p-1}}=\left(\sqrt{x_{\mathrm{c}}}\right)^{2 p-1} & \sum_{k=0}^{2 p-1}\left(\begin{array}{c}
2 p-1 \\
k
\end{array}\right) \Psi_{k} \Psi_{2 p-1-k} \\
+ & (2 p-1)\left(\sqrt{x_{\mathrm{c}}}\right)^{2 p-2} \sum_{k=0}^{p-2}\left(\begin{array}{c}
2 p-2 \\
2 k+1
\end{array}\right) \Psi_{2 k+2} \Psi_{2 p-2 k-2}+\mathrm{O}\left(\lambda^{p+1}\right) \\
\overline{\varepsilon_{n}^{2 p}} & =\left(\sqrt{x_{c}}\right)^{2 p} \sum_{k=0}^{p}\left(\begin{array}{c}
2 p \\
2 k
\end{array}\right) \Psi_{2 k} \Psi_{2 p-2 k}+\mathrm{O}\left(\lambda^{p+1}\right)
\end{aligned}
$$

where the $\Psi_{k}$ are given for $p \geqslant 2$ by

$$
\begin{aligned}
\Psi_{2 p-1}=A^{2 p-1} & \sum_{k=0}^{2 p-1}\left(\begin{array}{c}
2 p-1 \\
k
\end{array}\right) \overline{\varepsilon_{n-1}^{k}} \overline{\varepsilon_{n-1}^{2 p-1-k}} \\
& +(2 p-1) A^{2 p-2} B \sum_{k=0}^{p-2}\left(\begin{array}{c}
2 p-2 \\
2 k+1
\end{array}\right) \overline{\varepsilon_{n-1}^{2 k+2}} \overline{\varepsilon_{n-1}^{2 p-2-2 k}} \\
& -(2 p-1) A^{2 p-1} B \sum_{k=0}^{p}\left(\begin{array}{c}
2 p \\
2 k
\end{array}\right) \overline{\varepsilon_{n-1}^{2 k}} \overline{\varepsilon_{n-1}^{2 p-2 k}} \\
& \Psi_{2 p}=A^{2 p} \sum_{k=0}^{p}\left(\begin{array}{c}
2 p \\
2 k
\end{array}\right) \overline{\varepsilon_{n-1}^{2 k}} \overline{\varepsilon_{n-1}^{2 p-2 k}}
\end{aligned}
$$

and

$$
\Psi_{0}=1, \quad \Psi_{1}=2 A \overline{\varepsilon_{n-1}}-2 A \overline{B \varepsilon_{n-1}^{2}}, \quad \Psi_{2}=2 A^{2} \overline{\varepsilon_{n-1}^{2}} . \quad(29 e, f, g)
$$

\section{The fixed points and their exponents}

In $\S 4$, we have just seen how the moments of a distribution $P_{n-1}$ which fulfils the property (23) are transformed by one renormalisation step. We are now going to see that we can find a fixed point distribution which belongs to the class of the distributions which satisfy (23) when the specific heat exponent $\alpha_{\mathrm{p}}$ of the pure system is small and positive.

Let us choose a value of $q$ close to the value $q_{0}=4+2 \sqrt{2}$ where the exponent $\alpha_{p}$ vanishes (see equation (15)). For this value of $q$, the critical point $x_{c}(q)$ is close to $x_{c}\left(q_{0}\right)=3+2 \sqrt{2}$. Let us define $\Delta x$ by

$$
\Delta x=x_{c}(q)-(3+2 \sqrt{2}) \text {. }
$$

When $\Delta x$ is small, one has from (9)

$$
q-q_{0}=q-(4+2 \sqrt{2})=(1+\sqrt{2}) \Delta x+\mathrm{O}\left[(\Delta x)^{2}\right]
$$


and $\alpha_{\mathrm{p}}$ is small (see (14))

$$
\alpha_{\mathrm{p}}=[(10-7 \sqrt{2}) / \log 2] \Delta x+\mathrm{O}\left[(\Delta x)^{2}\right]
$$

When $\Delta x$ is small and positive, we can find two fixed distributions under the renormalisation transformation (23) which belong to the class of distributions which satisfy (23) with $\lambda \sim \Delta x$.

The first fixed distribution is the fixed point of the pure system. For all $p \geqslant 1$, the $\overline{\varepsilon^{\bar{p}}}$ of this distribution vanish

$$
\overline{\varepsilon^{\bar{p}}}=0 \text {. }
$$

The second fixed distribution corresponds to the random fixed point and the moments $\overline{\varepsilon^{p}}$ of this distribution can be obtained from $(24),(25),(26),(27)$ and (29) by writing that $\overline{\varepsilon_{n}^{p}}=\overline{\varepsilon_{n-1}^{p}}$ for all $p$.

Expanding the constants $A$ and $B$ given by (28) in powers of $\Delta x$, one obtains the moments of this random fixed point

$$
\begin{aligned}
& \bar{\varepsilon}=\frac{1}{7}(6+2 \sqrt{2}) \Delta x \\
& \overline{\varepsilon^{2}}=\frac{1}{7}(36+26 \sqrt{2}) \Delta x \\
& \overline{\varepsilon^{3}}=\frac{1}{49}(2736+1920 \sqrt{2})(\Delta x)^{2} \\
& \overline{\varepsilon^{4}}=\frac{1}{49}(7944+5616 \sqrt{2})(\Delta x)^{2} .
\end{aligned}
$$

One can also calculate from (29) higher moments of this fixed distribution and one finds that for $p \geqslant 2$

$$
\begin{aligned}
& \overline{\varepsilon^{2 p-r}}=\left(\overline{\frac{\varepsilon^{2}}{2}}\right)^{p} \frac{(2 p-1) !}{(p-1) !} \frac{4 p-p \sqrt{2}-4+2 \sqrt{2}}{(\sqrt{2}+1)^{2}} \\
& \overline{\varepsilon^{2 p}}=\frac{(2 p) !}{p !}\left(\overline{\varepsilon^{2}} \frac{p}{2}\right)^{p} .
\end{aligned}
$$

Clearly from (34b) and (35) one sees that this fixed distribution satisfies condition (23) and that it becomes narrower and narrower as $\Delta x \rightarrow 0$ (i.e. $\alpha_{p} \rightarrow 0$ ). One should notice that the first four moments of the fixed distribution (equations (34)) can be obtained from the four recursion relations (24) to (27) since condition (23) ensures us that higher moments would contribute only to higher orders in $\Delta x$.

At the pure as well as at the random fixed point, the critical exponents can be obtained from the eigenvalues of the matrix $M$ whose elements are

$$
M_{k, l}=\partial \overline{\varepsilon_{n}^{k}} / \partial \overline{\varepsilon_{n-1}^{l}} \text {. }
$$

The structure of this matrix is such that if one wants to calculate these eigenvalues up to the first order in $\Delta x$, it is enough to keep only the four recursion relations (24) to (27) and therefore one has to diagonalise a $4 \times 4$ matrix $M$. For $\Delta x$ small, these four recursion relations become

$$
\begin{aligned}
\overline{\varepsilon_{n}}=[2+(10- & 7 \sqrt{2}) \Delta x] \overline{\varepsilon_{n-1}}+\left[-\frac{1}{2}(3 \sqrt{2}-4)+\frac{1}{4}(109-77 \sqrt{2}) \Delta x\right] \overline{\varepsilon_{n-1}^{2}} \\
& +(6-4 \sqrt{2})\left(\overline{\varepsilon_{n-1}}\right)^{2}+\frac{1}{4}(113-80 \sqrt{2}) \overline{\varepsilon_{n-1}} \overline{\varepsilon_{n-1}^{2}} \\
& +\frac{1}{16}(734-519 \sqrt{2})\left(\overline{\varepsilon_{n-1}^{2}}\right)^{2}+\frac{1}{4}(17-12 \sqrt{2}) \overline{\varepsilon_{n-1}^{3}}+\frac{1}{16}(140-99 \sqrt{2}) \overline{\varepsilon_{n-1}^{4}}
\end{aligned}
$$




$$
\begin{aligned}
& \overline{\varepsilon_{n}^{2}}=[1+(10-7 \sqrt{2}) \Delta x] \overline{\varepsilon_{n-1}^{2}}+3\left(\overline{\varepsilon_{n-1}}\right)^{2}+\frac{1}{2}(36-25 \sqrt{2}) \overline{\varepsilon_{n-1}} \overline{\varepsilon_{n-1}^{2}} \\
& \quad+\frac{1}{8}(379-268 \sqrt{2})\left(\overline{\varepsilon_{n-1}^{2}}\right)^{2}+\frac{1}{2}(4-3 \sqrt{2}) \overline{\varepsilon_{n-1}^{3}}+\frac{1}{8}(51-36 \sqrt{2}) \overline{\varepsilon_{n-1}^{4}} \\
& \overline{\varepsilon_{n}^{3}}=\frac{9}{2} \overline{\varepsilon_{n-1}} \overline{\varepsilon_{n-1}^{2}}+\frac{1}{8}(108-75 \sqrt{2})\left(\overline{\varepsilon_{n-1}^{2}}\right)^{2}+\frac{1}{2} \overline{\varepsilon_{n-1}^{3}}+\frac{1}{8}(12-9 \sqrt{2}) \overline{\varepsilon_{n-1}^{4}} \\
& \overline{\varepsilon_{n}^{4}}=\frac{9}{4}\left(\overline{\varepsilon_{n-1}^{2}}\right)^{2}+\frac{1}{4} \overline{\varepsilon_{n-1}^{4}} .
\end{aligned}
$$

At the pure fixed point, one finds for the first two eigenvalues $\lambda_{1}^{\text {pure }}$ and $\lambda_{2}^{\text {pure }}$ of the matrix $M$ to first order in $\Delta x$

$$
\lambda_{1}^{\text {pure }}=2+(10-7 \sqrt{2}) \Delta x, \quad \lambda_{2}^{\text {pure }}=1+(10-7 \sqrt{2}) \Delta x .
$$

The first eigenvalue has an eigenvector $(1,0,0,0)$ whereas the second eigenvalue has the eigenvector $\left(\frac{1}{2}(3 \sqrt{2}-4)+\frac{1}{4}(77 \sqrt{2}-109) \Delta x, 1,0,0\right)$.

One sees clearly in $(38 b)$ that when $\Delta x$ becomes positive, the second eigenvalue becomes larger than 1 and disorder becomes relevant according to the Harris (1974) criterion.

If $\mu_{1}$ and $\mu_{2}$ are the two scaling fields for the first and the second eigenvalues, the singular part $f_{\text {sing }}$ of the free energy satisfies

$$
f_{\text {sing }}\left(\mu_{1}, \mu_{2}\right)=\frac{1}{4} f_{\text {sing }}\left(\lambda_{1}^{\text {pure }} \mu_{1}, \lambda_{2}^{\text {pure }} \mu_{2}\right)
$$

and therefore $f_{\text {sing }}$ has the following scaling form

$$
f_{\text {sing }}\left(\mu_{1}, \mu_{2}\right)=\mu_{1}^{2-\alpha_{p}} G\left(\mu_{2} / \mu_{1}^{\phi_{p}}\right)
$$

where the specific heat exponent $\alpha_{\mathrm{p}}$ and the cross-over exponent $\phi_{\mathrm{p}}$ are given by

$$
\begin{aligned}
& \alpha_{\mathrm{p}}=2-\frac{\log 4}{\log \left(\lambda_{1}^{\text {pure }}\right)}=\frac{10-7 \sqrt{2}}{\log 2} \Delta x+\mathrm{O}\left[(\Delta x)^{2}\right] \\
& \phi_{\mathrm{p}}=\frac{\log \left(\lambda_{2}^{\text {pure }}\right)}{\log \left(\lambda_{1}^{\text {pure }}\right)}=\frac{10-7 \sqrt{2}}{\log 2} \Delta x+\mathrm{O}\left[(\Delta x)^{2}\right] .
\end{aligned}
$$

We find as expected from the exponent relation of Harris that $\alpha_{\mathrm{p}}=\phi_{\mathrm{p}}$ at the pure fixed point (Kinzel and Domany 1981, Andelman and Berker 1984).

At the random fixed point, one finds for the first two eigenvalues

$$
\lambda_{1}^{\text {random }}=2+\frac{1}{7}(38-27 \sqrt{2}) \Delta x, \quad \lambda_{2}^{\text {random }}=1+(7 \sqrt{2}-10) \Delta x
$$

with eigenvectors $\left(1, \frac{1}{7}(16+24 \sqrt{2}) \Delta x, \quad \frac{1}{7}(108+72 \sqrt{2}) \Delta x, \quad 0\right)$ and $\left(\frac{1}{2}(3 \sqrt{2}-4)+\right.$ $\left.\frac{1}{28}(2781-1971 \sqrt{2}) \Delta x, 1, \frac{1}{7}(48+72 \sqrt{2}) \Delta x, \frac{1}{7}(216+156 \sqrt{2}) \Delta x\right)$.

As in the pure case, one can obtain from (42) the specific heat exponent $\alpha_{\mathrm{r}}$ and the cross over exponent $\phi_{\mathrm{r}}$ of the random fixed point

$$
\begin{aligned}
& \alpha_{\mathrm{r}}=\frac{38-27 \sqrt{2}}{7 \log 2} \Delta x+\mathrm{O}\left[(\Delta x)^{2}\right] \\
& \phi_{\mathrm{r}}=-\frac{10-7 \sqrt{2}}{\log 2} \Delta x+\mathrm{O}\left[(\Delta x)^{2}\right] .
\end{aligned}
$$

One should notice that when $\alpha_{\mathrm{p}}$ becomes positive, i.e. when $\Delta x$ becomes positive, the eigenvalue $\lambda_{2}^{\text {random }}$ is less than 1 and the random fixed point has only one relevant direction, the thermal one. 
For $\alpha_{\mathrm{p}}$ small, one can summarise the results (41) and (42) by expressing all exponents as functions of $\alpha_{\mathrm{p}}$ :

$$
\phi_{\mathrm{p}}=\alpha_{\mathrm{p}}=-\phi_{\mathrm{r}}, \quad \alpha_{\mathrm{r}}=-\frac{1}{7}(2 \sqrt{2}-1) \alpha_{\mathrm{p}} .
$$

One should notice that the specific exponent $\alpha_{\mathrm{r}}$ is negative (when $\alpha_{\mathrm{p}}>0$ ) but that at the random fixed point $\phi_{\mathrm{r}}$ is no longer equal to $\alpha_{\mathrm{r}}$ (Andelman and Berker 1984).

\section{The scaling fields}

In order to find the critical surface and the critical behaviour when $\alpha_{\mathrm{p}}$ vanishes, it is useful to introduce the two scaling fields $\mu_{1}$ and $\mu_{2}$ corresponding to the two eigenvalues $\lambda_{1}^{\text {pure }}$ and $\lambda_{2}^{\text {pure }}$ (Wegner 1976). Because we are working near $\Delta x=0$ where the eigenvalue $\lambda_{2}^{\text {pure }}=1$, the nonlinear part of the renormalisation transformation (37) cannot be absorbed into the scaling fields so that the renormalisation equations for $\mu_{1}$ and $\mu_{2}$ decouple (Wegner 1976). Instead, we could find two scaling fields, $\mu_{1}$ and $\mu_{2}$ such that the renormalisation transformations can be written

$$
\mu_{1}^{\prime}=\lambda_{1}^{\text {pure }} \mu_{1}-a \mu_{1} \mu_{2}, \quad \mu_{2}^{\prime}=\lambda_{2}^{\text {pure }} \mu_{2}-b \mu_{2}^{2}
$$

where $\mu_{1}^{\prime}$ and $\mu_{2}^{\prime}$ denote the values of the scaling fields after one renormalisation step.

Using formula (37) and assuming that the $\mu_{1}$ can be expanded in the following way:

$\mu=u_{1} \bar{\varepsilon}+\overline{\varepsilon^{2}}+u_{2} \Delta x \bar{\varepsilon}+u_{3}(\bar{\varepsilon})^{2}+u_{4} \bar{\varepsilon} \overline{\varepsilon^{2}}+u_{5}\left(\overline{\varepsilon^{2}}\right)^{2}+u_{6} \overline{\varepsilon^{3}}+u_{7} \overline{\varepsilon^{4}}$,

one can determine the constants $a$ and $b$ and all the constants $u$ except one. One finds that

$$
\begin{aligned}
& \mu_{1}^{\prime}=[2+(10-7 \sqrt{2}) \Delta x] \mu_{1}-(29 \sqrt{2}-41) \mu_{1} \mu_{2} \\
& \mu_{2}^{\prime}=[1+(10-7 \sqrt{2}) \Delta x] \mu_{2}-\frac{1}{2}(128 \sqrt{2}-181) \mu_{2}^{2}
\end{aligned}
$$

where $\mu_{1}$ and $\mu_{2}$ are given by

$$
\begin{gathered}
\mu_{1}=-(4+3 \sqrt{2}) \bar{\varepsilon}+\overline{\varepsilon^{2}}+\frac{1}{2}(-5+\sqrt{2}) \Delta x \bar{\varepsilon}+\frac{1}{2}(-3+2 \sqrt{2})(\bar{\varepsilon})^{2}+\frac{1}{8}(536-379 \sqrt{2})\left(\overline{\varepsilon^{2}}\right)^{2} \\
+\frac{1}{2}(4-3 \sqrt{2}) \overline{\varepsilon^{3}}+\frac{1}{2}(17-12 \sqrt{2}) \overline{\varepsilon^{4}}+D\left[-(4+3 \sqrt{2}) \bar{\varepsilon} \overline{\varepsilon^{2}}+\left(\overline{\varepsilon^{2}}\right)^{2}\right] \\
\mu_{2}=\overline{\varepsilon^{2}}-(\bar{\varepsilon})^{2}+(20 \sqrt{2}-28) \bar{\varepsilon} \overline{\varepsilon^{2}}+(4-3 \sqrt{2}) \overline{\varepsilon^{3}}+\frac{1}{2}(51-36 \sqrt{2}) \overline{\varepsilon^{4}}
\end{gathered}
$$

where the constant $D$ cannot be determined and remains arbitrary.

The equation of the critical surface, at $\operatorname{order}(\Delta x)^{2}$, is

$$
\begin{aligned}
\tilde{\mu}_{1}=-(4+3 \sqrt{2}) \bar{\varepsilon}+\overline{\varepsilon^{2}}+\frac{1}{2}(-5+\sqrt{2}) \Delta x \bar{\varepsilon}+\frac{1}{2}(-3+2 \sqrt{2})(\bar{\varepsilon})^{2} \\
\\
+\frac{1}{8}(536-379 \sqrt{2})\left(\overline{\varepsilon^{2}}\right)^{2}+\frac{1}{2}(4-3 \sqrt{2}) \overline{\varepsilon^{3}}+\frac{1}{2}(17-12 \sqrt{2}) \overline{\varepsilon^{4}} \\
=0
\end{aligned}
$$

where we have arbitrarily chosen $D=0$ in (48a).

One should notice that the constant $D$ has no effect in the equation of the critical surface because at order $(\Delta x)^{2}$, one can also write $(48 a)$ as a product:

$$
\mu_{1}=\left(1+D \overline{\varepsilon^{2}}\right) \tilde{\mu}_{1}
$$

and for narrow distributions $\mu_{1}=0$ this is equivalent to $\tilde{\mu}_{1}=0$. 
In $\$ 7$ we shall see how equations (47) determine the singularity of the free energy of the random system when $\Delta x=0$ i.e. when $\alpha_{\mathrm{p}}=0$.

\section{The singularity when $\alpha$ vanishes}

When $\alpha_{\mathrm{p}}$ vanishes (i.e. $\Delta x=0$ ) the scaling field $\mu_{2}$ that corresponds to randomness is marginal. Therefore, one expects logarithmic corrections to the critical behaviour. If we call $\mu_{1}(n)$ and $\mu_{2}(n)$ the values of the two scaling fields after $n$ renormalisations, from (47), we have

$$
\begin{aligned}
& \mu_{1}(n+1)=\left[2-a \mu_{2}(n)\right] \mu_{1}(n) \\
& \mu_{2}(n+1)=\mu_{2}(n)-b\left[\mu_{2}(n)\right]^{2}
\end{aligned}
$$

where $a$ and $b$ are two positive constants given by

$$
a=29 \sqrt{2}-41, \quad b=\frac{1}{2}(128 \sqrt{2}-181) .
$$

For narrow distributions, we can consider that the free energy $f\left(\left\{P_{0}\right\}\right)$ is a function of only the two scaling fields $\mu_{1}$ and $\mu_{2}$

$$
f\left(\left\{P_{0}\right\}\right)=f\left(\mu_{1}, \mu_{2}\right) .
$$

From (6), one can show that $f\left(\mu_{1}(n), \mu_{2}(n)\right)$ satisfies the following recursion relation

$$
\begin{aligned}
& f\left(\mu_{1}(n-1), \mu_{2}(n-1)\right) \\
& \quad=\frac{1}{4} f\left(\mu_{1}(n), \mu_{2}(n)\right)+\frac{1}{2} \iint \mathrm{d} x_{1} \mathrm{~d} x_{2} P_{n-1}\left(x_{1}\right) P_{n-1}\left(x_{2}\right) \log \left(x_{1}+x_{2}+q-2\right) .
\end{aligned}
$$

For narrow distributions $P_{n}$ which satisfy condition (23), one can expand the average on the right-hand side of equation (55) and one obtains

$$
\begin{aligned}
& f\left(\mu_{1}(n-1), \mu_{2}(n-1)\right) \\
& =\frac{1}{4} f\left(\mu_{1}(n), \mu_{2}(n)\right)+\frac{1}{2} \log \left(2 x_{\mathrm{c}}+q-2\right) \\
& +\frac{\overline{\varepsilon_{n-1}}}{2 x_{\mathrm{c}}+q-2}-\frac{1}{2} \frac{\overline{\varepsilon_{n-1}^{2}}+\left(\overline{\varepsilon_{n-1}}\right)^{2}}{\left(2 x_{\mathrm{c}}+q-2\right)^{2}}+\ldots
\end{aligned}
$$

where $\sqrt{x_{\mathrm{c}}}=1+\sqrt{2}$ and $q=\underline{4+2} \sqrt{2}$ since $\alpha_{\mathrm{p}}$ vanishes.

Knowing that $\overline{\varepsilon_{n-1}}$ and $\overline{\varepsilon_{n-1}^{2}}$ are related to $\mu_{1}(n-1)$ and $\mu_{2}(n-1)$ by $(48 a)$ and (48b), and that the $\mu_{i}(n)$ are transformed under one renormalisation step by (51) and (52), we want now to find the singular part of $f\left(\mu_{1}(0), \mu_{2}(0)\right)$ for small fixed $\mu_{2}(0)$ when $\mu_{1}(0) \rightarrow 0$. To do that, we can express $\overline{\varepsilon_{n-1}}$ and $\overline{\varepsilon_{n-1}^{2}}$ in terms of $\mu_{1}(n-1)$ and $\mu_{2}(n-1)$ by inverting (48):

$$
\begin{aligned}
& (4+3 \sqrt{2}) \overline{\varepsilon_{n-1}}=-\mu_{1}(n-1)+\mu_{2}(n-1)+\left[\mu_{1}(n-1)\right]^{2} \frac{2 \sqrt{2}-1}{2(4+3 \sqrt{2})^{2}}+O\left(\mu_{1} \mu_{2}, \mu_{2}^{2} \ldots\right) \\
& \overline{\varepsilon_{n-1}^{2}}=\mu_{2}(n-1)+\left[\mu_{1}(n-1)\right]^{2} \frac{1}{(4+3 \sqrt{2})^{2}}+O\left(\mu_{1} \mu_{2}, \mu_{2}^{2} \ldots\right) .
\end{aligned}
$$


The second derivative $f^{\prime \prime}\left(\mu_{1}(n), \mu_{2}(n)\right)$ of $f$ with respect to $\mu_{1}(n)$ obeys the following equation (see (51) and (56)):

$$
\begin{aligned}
f^{\prime \prime}\left(\mu_{1}(n), \mu_{2}(n)\right) & \\
= & \frac{1}{4}\left(2-a \mu_{2}(n)\right)^{2} f^{\prime \prime}\left(\mu_{1}(n+1), \mu_{2}(n+1)\right)+\frac{1}{2 x_{\mathrm{c}}+q-2} \frac{\mathrm{d}^{2} \overline{\varepsilon_{n}}}{\mathrm{~d} \mu_{1}(n)^{2}} \\
& -\frac{1}{2} \frac{1}{\left(2 x_{\mathrm{c}}+q-2\right)^{2}} \mathrm{~d}^{2} \frac{\left(\overline{\varepsilon_{n}^{2}}+\left(\overline{\varepsilon_{n}}\right)^{2}\right)}{\mathrm{d} \mu_{1}(n)^{2}} .
\end{aligned}
$$

Using (57) and replacing $q$ and $x_{c}$ by their values, one finds

$f^{\prime \prime}\left(\mu_{1}(n), \mu_{2}(n)\right)=\frac{1}{4}\left(2-a \mu_{2}(n)\right)^{2} f^{\prime \prime}\left(\mu_{1}(n+1), \mu_{2}(n+1)\right)+\frac{1}{4}(\sqrt{2}-1)^{9}$.

When $\mu_{2}(0)$ is small, one can integrate the recursion relations (51) and (52):

$$
\begin{aligned}
\mu_{2}(n) & =\mu_{2}(0) /\left(1+b \mu_{2}(0) n\right) \\
\mu_{1}(n) & =\mu_{1}(0) \prod_{k=0}^{n-1}\left(2-a \frac{\mu_{2}(0)}{1+b \mu_{2}(0) k}\right) \\
& \simeq 2^{n} \mu_{1}(0)\left(1+n b \mu_{2}(0)\right)^{-a / 2 b} .
\end{aligned}
$$

Near the fixed point $\mu_{1}(0)=\mu_{2}(0)=0$, the second derivative $f^{\prime \prime}$ may be written:

$$
f^{\prime \prime}\left(\mu_{1}(0), \mu_{2}(0)\right)=\frac{1}{4}(\sqrt{2}-1)^{9} \sum_{n=0}^{n_{c}} \frac{1}{4^{n}} \prod_{i=1}^{n}\left(2-\frac{a \mu_{2}(0)}{1+b \mu_{2}(0) i}\right)^{2}
$$

where $n_{\mathrm{c}}$ is the value of $n$ where $\mu_{1}(n)$ becomes of order 1 and therefore where the inhomogeneous term in equation (59) starts to be different from $\frac{1}{4}(\sqrt{2}-1)^{9}$.

$$
n_{\mathrm{c}} \sim \log \left(1 / \mu_{1}(0)\right) / \log 2 .
$$

For $\mu_{2}(0)$ small, one can replace the sum (62) by an integral. We find

$$
f^{\prime \prime}\left(\mu_{1}(0), \mu_{2}(0)\right)=\frac{(\sqrt{2}-1)^{9}}{4 \mu_{2}(0)} \frac{1}{(b-a)}\left[\left(1+b \mu_{2}(0) n_{c}\right)^{1-a / b}-1\right] .
$$

It can be checked that formulae (64) with $n_{\mathrm{c}}$ given by (63) solves equation (59). From (53), (63) and (64), one finds

$f^{\prime \prime}\left(\mu_{1}(0), \mu_{2}(0)\right)=\frac{7-5 \sqrt{2}}{2 \mu_{2}(0)}\left[\left(1+\mu_{2}(0) \frac{128 \sqrt{2}-181}{2 \log 2} \log \left(\frac{1}{\mu_{1}(0)}\right)\right)^{(1-2 \sqrt{2}) / 7}-1\right]$.

We see that for $\mu_{2}(0) \neq 0$, that $f^{\prime \prime}$ has a logarithmic singularity to a negative power when the temperature $T$ approaches the transition temperature $T_{\mathrm{c}}$.

$$
f_{\text {sing }}^{\prime \prime} \sim\left[\log \left(T-T_{\mathrm{c}}\right)\right]^{(1-2 \sqrt{2}) / 7} \text {. }
$$

Therefore a weak disorder is sufficient to suppress the divergence of the specific heat. At the transition temperature the specific heat has a finite maximum which increases like the inverse of the width of the distribution $\left(\overline{\varepsilon^{2}} \sim \mu_{2}(0)\right)$ when the distribution becomes narrower and narrower.

It can be checked that the limit $\mu_{2}(0) \rightarrow 0$ in (65) recovers the pure system logarithmic singularity (18). 


\section{Essential singularities}

We would like to discuss now the possibility of essential singularities in the free energy due to the disorder.

Consider a distribution of the interactions $x$ whose support is the interval $\left[x_{\min }, x_{\max }\right]$ with $x_{\max }>x_{\min }>x_{\mathrm{c}}$ where $x_{\mathrm{c}}$ is the critical temperature of the pure system. More precisely, $\left[x_{\min }, x_{\max }\right]$ is the smallest interval which contains the support of the distribution. In this section, we will suggest that the free energy has an essential singularity of the following form as $x_{\min } \rightarrow x_{\mathrm{c}}$.

$$
f_{\text {sing }} \sim \exp \left(-\frac{\text { constant }}{\left(x_{\min }-x_{c}\right)^{2-\alpha_{p}}}\right)
$$

where $\alpha_{p}$ is the specific heat exponent of the pure system.

We expect also a similar formula involving $x_{\max }$ to be valid when $x_{\min }<x_{\max }<x_{\mathrm{c}}$ and in the limit $x_{\max } \rightarrow x_{\mathrm{c}}$.

To obtain formula (66) we shall need to use two principal approximations. The first one consists in replacing the renormalisation transformation $R$ by a linearised transformation. The second approximation consists in replacing the sum over $n(6)$ by an integral over $n$.

In the neighbourhood of the fixed point $x_{c}$ of the pure system, let us replace the renormalisation transformation $R$ given in formula (3) by its linearised form:

$$
\varepsilon_{n}=x_{n}-x_{\mathrm{c}}=\frac{1}{4} T^{\prime}\left(x_{\mathrm{c}}\right) \sum_{i=1}^{4}\left(x_{n-1}^{(i)}-x_{\mathrm{c}}\right)=\frac{1}{4} T^{\prime}\left(x_{\mathrm{c}}\right) \sum_{i=1}^{4} \varepsilon_{n-1}^{(i)} \text {. }
$$

Now that the renormalisation map is linear, we can calculate the renormalised distributions $P_{n}(x)$ knowing the distribution $P_{0}(x)$. To simplify the discussion, we shall consider a distribution $P_{0}(x)$ which is the sum of two delta functions

$$
P_{0}(x)=\frac{1}{2}\left[\delta\left(x-x_{\min }\right)+\delta\left(x-x_{\max }\right)\right] .
$$

However, we believe that (66) should remain valid for more general distributions $P_{0}(x)$. The probability $Q_{n}(u)$ d $u$ that $x$ belongs to the interval $I$ defined by

$$
\begin{aligned}
& I=\left[x_{\mathrm{c}}+\left(T^{\prime}\right)^{n}\left(x_{\min }(1-u)+x_{\max } u-x_{\mathrm{c}}\right),\right. \\
& \left.x_{\mathrm{c}}+\left(T^{\prime}\right)^{n}\left(x_{\min }(1-u-\mathrm{d} u)+x_{\max }(u+\mathrm{d} u)-x_{\mathrm{c}}\right)\right]
\end{aligned}
$$

has for large $n$ the following form

$$
Q_{n}(u)=\left(h^{\prime \prime}(u) / 2 \pi\right)^{1 / 2} 2^{n} \exp \left[4^{n} h(u)\right]
$$

where $h(u)$ is a function defined on the interval ]0, 1 [ which is negative on the whole interval ]0, 1 [ except in one point $\left(u=\frac{1}{2}\right)$ where $h$ vanishes with a quadratic maximum. For $P_{0}(x)$ given by $(68)$, one has

$$
h(u)=-u \log u-(1-u) \log (1-u)-\log 2 .
$$

We can use the formula (6) to obtain the singular part of the free energy. Since in the sum (6) the singularities come only from the contribution of large $n$ 's, we can use the asymptotic form (70) and we get

$$
f\left(\left\{P_{0}\right\}\right)=\int_{0}^{1} \mathrm{~d} u F(u)
$$


with

$F(u)=\sum_{n=0}^{\infty} Q_{n+1 / 2}(u) \frac{1}{4^{n}} \frac{1}{2} \log \left[2 x_{\mathrm{c}}+q-2+2\left(T^{\prime}\right)^{n}\left(x_{\min }-x_{\mathrm{c}}+u\left(x_{\max }-x_{\min }\right)\right)\right]$.

We have used the fact that if $x_{1}$ and $x_{2}$ are distributed according to a distribution which is represented by $Q_{n}(u)$, then the sum $x_{1}+x_{2}$ has a distribution represented by $Q_{n+1 / 2}(u)$.

Now the mathematical problem which should be solved is to show that $f\left(\left\{P_{0}\right\}\right)$ given by (72) and (73) has an essential singularity of the form (66) as $x_{\min } \rightarrow x_{c}$ with $x_{\max }-x_{\min }$ fixed. $\tilde{F}(u)$.

Let us now do our second approximation and replace the sum $F(u)$ by an integral

$\tilde{F}(u)=\int_{0}^{\infty} \mathrm{d} n Q_{n+1 / 2}(u) \frac{1}{4^{n}} \frac{1}{2} \log \left[2 x_{\mathrm{c}}+q-2+2\left(T^{\prime}\right)^{n}\left(x_{\min }-x_{\mathrm{c}}+u\left(x_{\max }-x_{\min }\right)\right)\right]$.

Except for the value of $u$ for which $h(u)$ vanishes, one can show that $\tilde{F}(u)$ has the following singularity when $\delta=x_{\min }-x_{\mathrm{c}}+u\left(x_{\max }-x_{\min }\right) \rightarrow 0$ keeping $u$ fixed:

$$
\tilde{F}_{\text {sing }}^{(u)} \sim \exp -\left[\frac{\text { constant }}{\left(x_{\min }-x_{c}+u\left(x_{\max }-x_{\min }\right)\right)^{2-\alpha_{p}}}\right] .
$$

To justify (75) from (74), one can expand the logarithm in (74) in powers of $\delta$. Since the dependence on $n$ is simple, one can integrate the high order terms in this expansion over $n$ using a saddle point method. One finds a zero radius of convergence of the series in $\delta$. Then using the Borel transform, one gets (75).

When one integrates over $u$ the neighbourhood of $u=0$ should give the singularity (66).

The justifications of (66) given in this section are clearly not sufficient to establish the presence of essential singularities when one edge of the distribution approaches the critical point of the pure system (Griffiths 1969). We believe that the linearisation of the renormalisation transformation should not change the form of the singularity. However replacing the sum by an integral or integrating over $u$ could change the nature of the singularity. Therefore we think that it would be worthwhile to try and find the singularities of the function $f$ given by (72) and (73).

\section{Conclusion}

We have seen in this paper that one can make a rather precise study of the critical properties of a spin model with random interactions on a hierarchical lattice. As expected from the Harris criterion, we have found that a new fixed point appears when $\alpha_{p}$ becomes positive and we have obtained the exponents of this random fixed point perturbatively when $\alpha_{\mathrm{p}}$ is small. We think that it would be interesting. to go to higher orders in the expansion of these exponents in order to compare these exponents with the numerical estimations of Andelman and Berker (1984). We have also seen that one can find the logarithmic corrections for $\alpha_{p}=0$.

We think that it would be very interesting to pursue the study of disordered systems on hierarchical lattices. Because the pure system on hierarchical lattices can be solved in a few lines, one can go much further in the study of disordered systems on such 
lattices than on usual Bravais lattices and answer more delicate questions. We think, in particular, that it should be very interesting to prove the existence of the essential singularities described in $\$ 8$ and to study the analytic properties of the free energy when $x_{\min }<x_{\mathrm{c}}<x_{\max }$.

\section{Acknowledgments}

We would like to thank $\mathrm{H}$ Flyvbjerg for an illuminating discussion. This work was partly supported by a Research Fellowship from the Royal Society.

\section{References}

Andelman D and Berker A N 1984 Phys. Rev. B 292630

Benyoussef A and Boccara N 1982 J. Phys. C: Solid State Phys. 151381

Berker A N and Ostlund S 1979 J. Phys. C: Solid State Phys. 124961

Burkhardt T W and Van Leeuwen J M J 1982 Real space renormalization vol 30 (Berlin: Springer)

Collet P, Eckmann J P, Glaser V and Martin A 1984 Commun. Math. Phys. 94353

Derrida B, de Seze L and Itzykson C 1983 J. Stat. Phys. 33559

Derrida B, Itzykson C and Luck J M 1984 Commun. Math. Phys. 94115

Griffiths R B 1969 Phys. Rev. Lett. 2317

Griffiths ·R B and Kaufman M 1982 Phys. Rev. B 265022

Harris A B 1974 J. Phys. C: Solid State Phys. 71671

Jayaprakash C, Riede E K and Wortis M 1978 Phys. Rev. B 182244

Kadanoff L P 1976 Ann. Phys., NY 100359

Kaufman M and Andelman D 1984 Phys. Rev. B 294010

Kaufman M and Griffiths R B 1981 Phys. Rev. B 24496

— 1983 Phys. Rev. B 265282

Kinzel W and Domany E 1981 Phys. Rev. B 233421

McKay S R and Berker A N 1984 Phys. Rev. B 291315

Melrose J R 1983 J. Phys. A: Math. Gen. 161041,3077

Migdal A A 1976 Sov. Phys.-JETP 42743

Southern B W, Young A P and Pfeuty P 1979 J. Phys. C: Solid State Phys. 12683

Wegner F 1976 in Phase Transitions and Critical Phenomena vol 6, ed C Domb and M S Green (New York: Academic)

Yeomans J M and Stinchcombe R B 1979 J. Phys. C: Solid State Phys. 12347 